\title{
Never too late to discover some extra thyroid tissue
}

\author{
Wessling $\mathrm{A}^{1}$, Aragüés JM ${ }^{1,2}$, Ferreira $\mathrm{F}^{1}$, Carmo $\mathrm{I}^{1,2}$ \\ ${ }^{1}$ Endocrinology Department of Santa Maria University Hospital, Lisbon, Portugal \\ ${ }^{2}$ Medical School, Lisbon University, Lisbon, Portugal
}

\section{Introduction}

The thyroid gland is the first endocrine gland that appears during embryonic development, approximately on the $24^{\text {th }}$ day of gestation. Ectopic thyroid is a rare entity, resulting from developmental defects at early stages of thyroid embryogenesis, in it's normal migration process from the floor of the primitive foregut to the pretracheal location. It's prevalence is 1 per 100000 - 300000 in general population and 1 per 4000-8000 in patients with thyroid disease. This condition is more common in females, Asians and may occur at any age, although it's most common at younger ages. The mean age of presentation is 40,5 years.

The exact mechanisms responsible are still unclear but many transcription factors appear to play a key role such as TITF1/NKX2-1, PAX 8, HHEX and FOXE 1. Thyroid ectopy may also have familial/hereditary background: heterozygous mutations in PAX 8 and expression of the Thyroid-Per-Oxidase and Thyroglobulin Genes.

The most frequent (about 90\%) location of ectopic thyroid tissue is at the base of the tongue and in those, 70 $75 \%$ of cases it's the only thyroid tissue present. The most common symptoms are related to the growth of lingual thyroid (dysphagia, dysphonia, sleep apnea or even respiratory obstruction and hemorrhage) and hypothyroidism, specially in the absence of orthotopic thyroid.

\section{Clinical case}

We present a case report from a asymptomatic caucasian male, 82 years, referred to our practice with subclinical hypothyroidism diagnosed at routine clinical evaluation. His blood tests showed an elevated TSH $(11,48 \mathrm{uU} / \mathrm{mL})$ with normal levels of free T4 $(0,98 \mathrm{ng} / \mathrm{dL})$. The ultrasound scan showed a decreased size thyroid gland and head and neck CT scan, ordered because for other clinical reasons showed a $25 \mathrm{~mm}$ sublingual thyroid gland, confirmed by scintigraphy (Figure 1).

He was put on Levothyroxine 50ug daily and 4 months later, had, normal thyroid tests. He also did a Laryngoscopy which confirmed a well defined sublingual mass (Figure 2).

The dose of Levothyroxine was maintained and, as the patient kept asymptomatic no other treatment was needed.

\section{Conclusion}
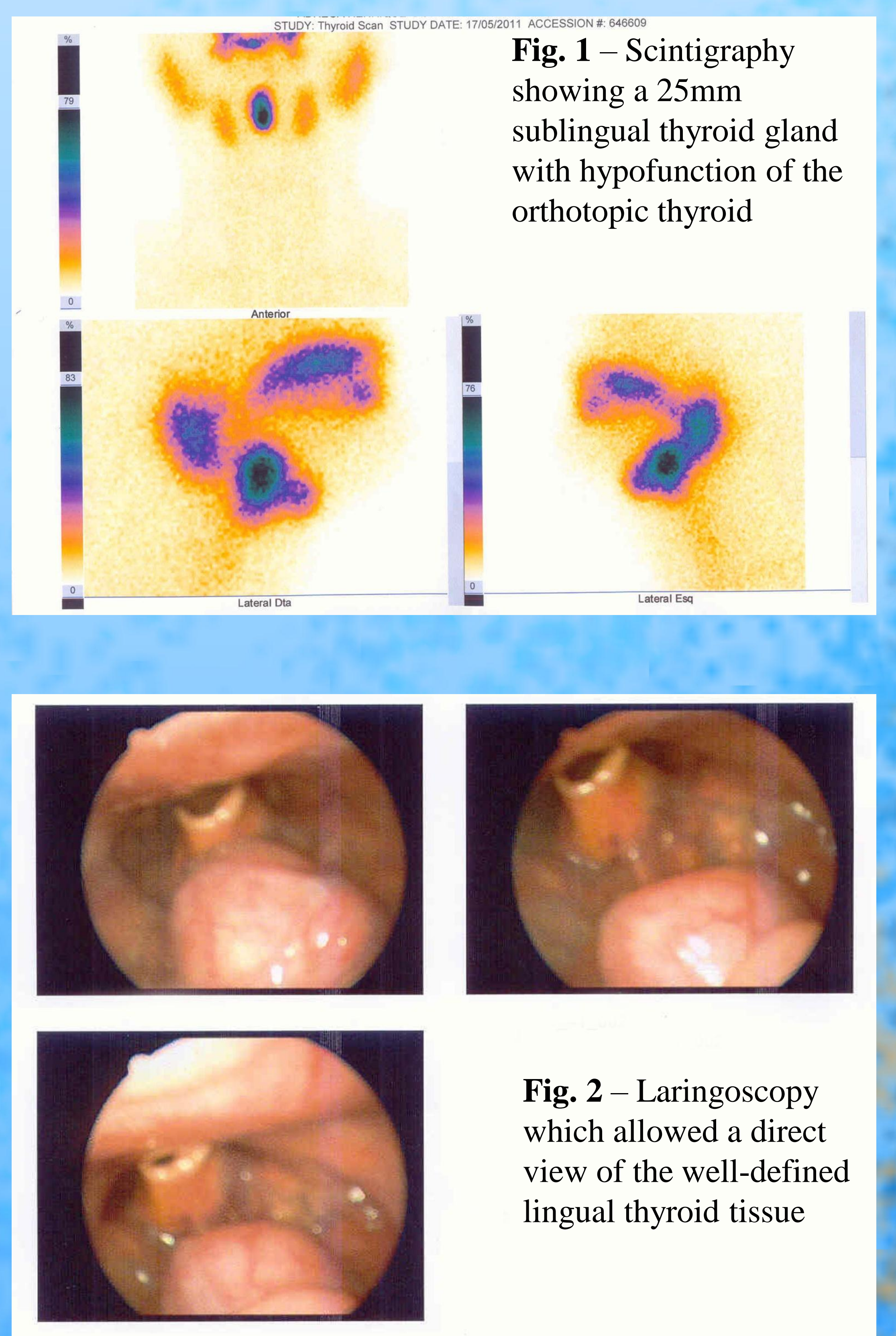

Fig. 2 - Laringoscopy which allowed a direct view of the well-defined lingual thyroid tissue

This is a case of a Caucasian male with a rare congenital condition, more common in Asian women. In 70-75\% the lingual thyroid is the only thyroid tissue present, so this patient belongs to the minority group who also have a orthotopic thyroid (although atrophic), accounting for only $25-30 \%$ of these cases. This is probably the main reason why he kept asymptomatic for most of his life, until the age of 82 (mean age of diagnosis is around 40,5 years) and his clinical presentation consisted only in an asymptomatic subclinical hypothyroidism.

scintigraphy is the most important tool to detect ectopic thyroid tissue, although there is a possibility of false positive as a result of normal or abnormal uptake in head and neck. There is no consensus about the optimal therapeutic management, but surgery is usually indicated for severe, incapacitating or even life threatening symptoms. For asymptomatic patients or the ones with mild symptoms and hypothyroidism, levothyroxine replacement may be effective leading to considerable mass reduction. Doctors must also keep in mind that thyrotoxicosis and thyroid tumors can also occur in the ectopic tissue as in the orthotopic thyroid gland.

So, in clinical practice we should be careful precluding any condition just based on probability, although the clinician should always take into account the rarity of this entity and differentiate it from other masses in neck. 